\title{
Effect of piracetam on one-way active avoidance in rats with medial thalamic lesions
}

\author{
PATRICIA A. ABBOTT and LARRY W. MEANS \\ East Carolina University, Greenville, North Carolina 27834
}

\begin{abstract}
Rats sustaining either medial thalamic electrolytic lesions or sham operations were injected with either piractam $(100 \mathrm{mg} / \mathrm{kg})$, a drug previously shown to enhance acquisition, or saline for 3 consecutive days prior to being tested for shock-flinch threshold and one-way active avoidance. The animals with medial thalamic lesions were found to make fewer avoidances than the sham-operated animals. Piracetam was shown neither to alleviate the lesion-induced avoidance deficit nor to facilitate acquisition in the sham-operated animals. No differences were observed in shock-flinch threshold.
\end{abstract}

Rats with relatively large lesions centered in the dorsomedial nucleus of the thalamus (DMT) have been found to be deficient on acquisition of one-way active avoidance (Tigner, 1972; Vanderwolf, 1964, 1966, 1967; Wuensch \& Means, 1976). Several treatments, including septal lesions (Vanderwolf, 1964), warm-up period (Vanderwolf, 1966), electroconvulsive shock (Vanderwolf, 1968), and chlorpromazine injections (Wuensch \& Means, 1976) have been found to partially alleviate the DMT lesion-induced active avoidance deficit. It has been suggested that all of the above treatments improve the performance of DMT-lesioned rats in avoidance conditioning by decreasing the tendency to behaviorally freeze when shocked. Further, it has been shown that amphetamine injections decrease the freezing behavior of DMT-lesioned rats in a two-choice discrimination task, but they do not improve choice performance (Wuensch, Broom, Means, \& Harris, 1978). Thus, several treatments have been found to decrease the freezing behavior of DMT-lesioned rats, but no treatment has been found that directly affects the acquisition process.

Piracetam (2-pyrrolidone acetamide) has been proposed as a learning-facilitation drug (Giurgea, Note 1). In humans, it has been shown to alleviate some of the symptoms of senility (Stegnik, 1972). In rats, piracetam has been found to facilitate acquisition of a lightsignaled active avoidance in a Y maze (Wolthuis, 1971) and to reduce errors and escape latency in a four-choice water-filled maze (Giurgea \& Mouravieff-Lesiusse, 1972). It has also been shown to protect against the amnestic effects of electroconvulsive shock (Sara \& David-Remacle, 1974) and hypoxia (Giurgea, Lefevre, \& Lescrenier, 1971; Sara \& Lefevre, 1972). Thus piracetam has been shown to facilitate acquisition or protect retention under conditions of hypoxia, but it does not affect general activity levels.

This paper is based on an MA thesis by P. A. Abbott. Reprint requests should be sent to Larry W. Means, East Carolina University, Greenville, North Carolina 27834. Piracetam was furnished by UCB, Brussels, Belgium.
The present study was conducted to determine if piracetam can reduce a DMT lesion-induced acquisition deficit and/or facilitate acquisition in sham-operated animals.

\section{METHOD}

\section{Subjects}

A total of 41 individually housed, male, Long-Evans rats from the East Carolina University colony were used. All rats were given food and water ad lib.

The study involved four groups. Eight rats received lesions centered on the DMT and were treated with piracetam (Group D-P). Group S-P $(n=12)$ received sham operations and treatment with piracetam. Group D-S $(n=9)$ received lesions cntered on the DMT and saline. Group S-S $(n=12)$ received sham operations and saline.

\section{Procedure}

Surgery. Surgery was done under clean conditions; sodium pentobarbital was used as anesthesia (Nembutal, $50 \mathrm{mg} / \mathrm{kg}$ ) and $.2 \mathrm{cc}$ of Bicillin was administered to prevent infection. Subjects in Group D-P and Group D-S received bilateral lesions centered on the DMT. Animals were placed in the stereotaxic instrument with the tooth bar $5.0 \mathrm{~mm}$ above the intraaural line. Coordinates used were $1.2 \mathrm{~mm}$ posterior to bregma, $1.0 \mathrm{~mm}$ lateral to midline, and $6.2 \mathrm{~mm}$ ventral from the top surface of the skull. Lesions were made by passing $1.8 \mathrm{~mA}$ anodal $\mathrm{dc}$ for $12 \mathrm{sec}$, with a stainless steel electrode insulated with epoxylite except for the tip. Subjects in Groups S-P and S-S had electrodes lowered but received no current.

\section{Injections}

After the animals were allowed to recuperate for at least 3 weeks, the appropriate drug-injection procedure was initiated. Animals in Groups D-P and S-P received IP injections of piracetam $(100 \mathrm{mg} / \mathrm{kg})$ in distilled water on 2 successive days prior to testing and $30 \mathrm{~min}$ prior to the start of behavioral testing, as recommended by Giurgea (Note 2). Rats in Groups D-S and S-S received IP injections of .9\% isotonic saline on the same time schedule.

Behavioral testing. Shock-flinch threshold and one-way active avoidance were conducted in the same avoidance box (Lafayette Instrument Company, Model A 586). The avoidance box consisted of a shock compartment with a grid scrambler (Model E 1064GS). A warning tone could be sounded via a cone speaker mounted on the wall of the shock compartment opposite the safe compartment.

Shock-flinch threshold was evaluated to determine any 
differences among the groups in shock reactivity. Each animal was placed in the shock compartment of the avoidance box with the sliding door closed. A series of $35.5-\mathrm{sec}$ presentations of 7 shock levels $(.10, .13, .16, .20, .25, .30$, and $.40 \mathrm{~mA})$ was given randomly to each animal, with each level presented five times. The intertrial interval (ITI) was random, with a mean of 15 sec. Two observers, naive with respect to the animal's group, independently rated the response of each animal. A flinch, defined as a sudden movement of head or body occurring at the time of the shock onset, was recorded as present or not present (Gispen, Van Wimersma Greidanus, \& de Wied, 1970). The shock-flinch threshold for each rat was defined as the point at which the probability of response was $50 \%$.

Immediately following the evaluation of shock-flinch threshold, avoidance training was begun. Each animal received 100 trials in one training session. The ITI was varied randomly between $5 \mathrm{sec}$ and $30 \mathrm{sec}$, with a mean ITI of $17 \mathrm{sec}$. The CS consisted of a warning tone and the simultaneous opening of the sliding door to the safe compartment. The US, a .8-mA shock, was presented $15 \mathrm{sec}$ after the presentation of the tone if the rat had not jumped to the safe compartment. The shock and tone terminated simultaneously when the rat jumped to the safe compartment or when the rat had received $45 \mathrm{sec}$ of shock. If the rat failed to avoid or escape the shock, the door was closed when the shock and the tone terminated. The door remained closed for $5-20 \mathrm{sec}$ and was reopened simultaneously with the presentation of the next tone. If the rat escaped or avoided the shock, it was allowed to remain in the safe compartment for the remainder of the trial or for at least $30 \mathrm{sec}$. Number of avoidances and avoidance latencies were recorded

Histology. At the conclusion of behavioral testing, all animals in Groups D-S and D-P were sacrificed with an overdose of sodium pentobarbital and then were intracardially perfused with saline followed by buffered formalin. Brains were extracted, fixed in buffered formalin, and sectioned at 50 microns while frozen. Every fourth section was stained with thionin and compared to plates from Pellegrino and Cushman's (1967) atlas to determine the locus and the extent of the lesions.

\section{RESULTS}

\section{Anatomical}

All 17 animals receiving medial thalamic lesions sustained bilateral damage to the DMT and surrounding nuclei similar to the damage reported in earlier studies (Means, Hunt, Whiteside, \& Bates, 1973; Wuensch et al., 1978).

\section{Behavioral}

Shock-flinch threshold. Mean shock-flinch threshold and standard error of the mean (SEM) for each group are shown in Table 1. A two-way analysis of variance showed no significant differences in shock-flinch threshold among the groups.

Three pairs of raters determined the presence or absence of flinch responses. The interrater reliability for each pair of raters was determined using a phi coefficient. The overall phi coefficient was $.92\left[\chi^{2}(1)=\right.$ $1,214.58, \mathrm{p}<.01]$.

Shock-flinch threshold did not correlate with the number of avoidances for all groups combined [rho(39) $=-.20]$, nor were correlations significant for Group S-P
Table 1

Mean and Standard Error of the Mean for Shock-Flinch Threshold (in Milliamperes), Number of Avoidances, and Avoidance Latency (in Seconds)

\begin{tabular}{|c|c|c|c|c|c|c|c|}
\hline \multirow[b]{2}{*}{ Lesion } & \multirow[b]{2}{*}{ Drug } & \multicolumn{2}{|c|}{$\begin{array}{c}\text { Shock } \\
\text { Threshold }\end{array}$} & \multicolumn{2}{|c|}{$\begin{array}{l}\text { Number of } \\
\text { Avoidances }\end{array}$} & \multicolumn{2}{|c|}{$\begin{array}{c}\text { Avoidance } \\
\text { Latency }\end{array}$} \\
\hline & & $\mathbf{M}$ & SEM & $\mathbf{M}$ & SEM & M & SEM \\
\hline Sham & Piracetam & .18 & .0162 & 91.92 & 1.98 & 2.4 & .38 \\
\hline Sham & Saline & .15 & .0092 & 90.25 & 2.06 & 1.8 & .30 \\
\hline DMT & Piracetam & .15 & .0170 & 81.50 & 5.83 & 2.5 & .71 \\
\hline DMT & Saline & .17 & .0133 & 79.00 & 6.52 & 2.2 & .34 \\
\hline
\end{tabular}

$[\operatorname{rho}(10)=-.04]$, Group S-S $[\operatorname{rho}(10)=-.11]$, or Group D.P $[\operatorname{rho}(6)=-.32]$. However, there was a significant negative correlation between shock-flinch threshold and number of avoidances in Group D-S $[\mathrm{rho}(7)=-.81, \mathrm{p}<.05]$.

One-way active avoidance. Mean total avoidances and SEM for each group are given in Table 1. A three-way (lesion by drug by blocks of 10 trials) analysis of variance revealed that rats with medial thalamic lesions made significantly fewer avoidances than sham-operated animals $[F(1,37)=6.77, p<.025]$, but that the total number of avoidance responses did not differ significantly between the piracetam-treated and the salinetreated animals. Also, the interaction between drug and lesion groups was not significant.

The mean number of avoidances for each group for each block of 10 trials is presented in Figure 1. There was a significant trials effect $[\mathrm{F}(9,333)=38.57$, $\mathrm{p}<.001]$. A Newman-Keuls follow-up revealed that all groups improved significantly from the first 10 trials to the second 10 trials. There were no other significant changes over trials. A two-way analysis of variance of a logarithmic transformation of the latency scores (see Table 1) revealed no significant differences among the groups.



Figure 1. Mean number of avoidances for blocks of $\mathbf{1 0}$ trials. 


\section{DISCUSSION}

The results of the study fail to support the notion that pirace tam either facilitates acquisition in sham-operated animals or reduces a DMT lesion-induced deficit. The results are at variance with studies reporting facilitation of avoidance acquisition following treatment with piracetam (Giurgea et al., 1971; Sara \& David-Remacle, 1974; Wolthuis, 1971). The most obvious explanation of the fact that piracetam did not partially reverse the DMT lesion-induced deficit is that the mechanism of piracetam facilitation is unrelated to the disruptive anatomical and biochemical effects of DMT lesions. It has been suggested that piracetam increases oxygen availability (Giurgea et al., 1971; Pede, Schimpfesse1, \& Crokaert, 1971; Sara \& David-Remacle, $1974)$ and thus protects subjects from hypoxia-induced amnesia or facilitates acquisition. Why lesions of the DMT produce deficits on learning tasks is not understood, but this may be due to changes in attentional processes.

One further factor that may have influenced the lack of piracetam effect is the finding that, in the rat, piracetam reaches its maximum strength in the brain $2 \mathrm{~h}$ following injection (Ostrowski, Keil, \& Schraven, 1975). However, many studies have reported piracetam facilitation $30 \mathrm{~min}$ following injection (Giurgea et al., 1971; Sara \& David-Remacle, 1974; Sara \& Lefevre, 1972; Wolthuis, 1971).

While this study appears to be inconsistent with the results of Giurgea and Mouravieff-Lesiusse (1972) and Wolthuis (1971), it is in total agreement with several efforts in our laboratory to demonstrate either facilitated acquisition or a diminished DMT lesion-induced deficit. In a study demonstrating a significant DMT lesion-induced deficit in brightness discrimination, piracetam was not found to alter the performance of either sham-operated or DMT-lesioned rats (Means \& Gaino, Note 3). In another study (Franklin \& Means, Note 4), while age was shown to produce deficits in both active and passive avoidance, piracetam was not shown to affect the behavior of young or old rats. Further, using the same avoidance apparatus used in the present study, we have shown that chlorpromazine (.5 or $1.0 \mathrm{mg} / \mathrm{kg}$ ) improves the acquisition of avoidance in DMTlesioned rats (Wuensch \& Means, 1976). Thus, we feel that the potential of piracetam to facilitate avoidance acquisition in normal subjects or to reverse a lesion-induced deficit is limited, at best.

In conclusion, medial thalamic lesions impair the acquisition of one-way avoidance in rats. Piracetam was not found to be effective in reducing the deficit resulting from medial thalamic lesions or to facilitate acquisition in sham-operated rats.

\section{REFERENCE NOTES}

1. Giurgea, C. Differential experimental definition of nootropic drugs. Nooanaleptics and nootropics. Symposium presented at the meeting of the Third International Congress of Psychosomatic Medicine, Rome, 1975.

2. Giurgea, C. Personal communication, 1975.

3. Means, L. W., \& Gaino, J. Manuscript in preparation, 1979.

4. Franklin, R. D., \& Means, L. W. Manuscript in preparation, 1979.

\section{REFERENCES}

Gispen, W. H., Van Wimersma Greidanus, T. B., \&
DE WIED, D. Effects of hypophysectomy and ACTH 1-10 on responsiveness to electric shock in rats. Physiology \& Behavior, 1970, 5, 143-146.

Giurgea, C., Defevre, D., Lescrenier, C., \& DavidRemaCle, L. Pharmacological protection against hypoxia induced amnesia in rats. Psychopharmacologia, 1971, 20, 160-168.

Giurgea, C., \& Mouravieff-Lesiusse, F. Effect facilitateur du piracetam sur un apprentissage répétitif chez le rat. Journal de Pharmacologie, 1972, 3, 17-30.

Means, L. W., Hunt, M. W., Whiteside, R. B., \& Bates, T. W. Deficient acquisition and retention of single-alternation go, no-go in rats with medial thalamic lesions. Physiological Psychology, 1973, 1, 287-291.

Ostrowski, V. J., Keil, M., \& Schraven, E. Autoradiographische Untersuchungen zur Vertailung von Piracetam-14c bei Ratte und Hund. Arzneimittel-Forschung, 1975, 25, 589-596. (Abstract)

Pede, J. P., Schimpfessel, L., \& Crokaert, R. The action of piracetam on the oxidative phosporylation. Société Belge de Biochimie, 1971, 79, 1036-1037.

Pellegrino, L. J., \& Cushman, A. J. A stereotaxic atlas of the rat brain. New York: Appleton-Century-Crofts, 1967.

Sara, S. J., \& David-Remacle, M. Recovery from electroconvulsive shock-induced amnesia by exposure to the training environment: Pharmacological enhancement by piracetam. Psychopharmacologia, 1974, 36, 59-66.

SarA, S. J., \& Lefevre, D. Hypoxia-induced amnesia in onetrial learning and pharmacological protection by piracetam. Psychopharmacologia, 1972, 25, 32-40.

Stegnik, A. The clinical use of piracetam, a new nootropic drug: The treatment of symptoms of senile involution. ArzneimForsch, 1972, 22, 975-977.

Tigne R, J. C. Impairment of one-way active avoidance in rats with habenular or dorsomedial thalamic lesions. Psychonomic Science, 1972, 27, 7-8.

VANDERWOLF, C. H. Effect of combined medial thalamic and septal lesions on active-avoidance behavior. Journal of Comparative and Physiological Psychology, 1964, 58, 31-37.

VANDERWOLF, C. H. Warm-up effects in the avoidance performance of rats with medial thalamic lesions. Animal Behaviour, $1966,14,425-429$.

VANDERWOLF, C. H. Effects of experimental diencephalic damage on food hoarding and shock avoidance behavior in the rat. Physiology \& Behavior, 1967, 2, 399-402.

VANDERWOLF, C. H. Recovery from large medial thalamic lesions as a result of electroconvulsive therapy. Journal of Neurology, Neurosurgery and Psychiatry, 1968, 31, 67-72.

Wolthuis, O. L. Experiments with UCB 6215, a drug which enhances acquisition in rats: Its effects compared with those of metamphetamine. European Journal of Pharmacology, 1971, 16, 283-297.

Wuensch, K. L., Broome, B., Means, L. W., \& Harris, E. C. Effects of dorsomedial thalamic lesions and amphetamine on acquisition and retention of a visual pattern discrimination escape task. Physiological Psychology, 1978, 6, 288-293.

Wuensch, K. L., \& Means, L. W. Chlorpromazine reduces avoidance performance deficit in rats with dorsomedial thalamic lesions. Bulletin of the Psychonomic Society, 1976, 8, 439-440. 